\title{
Role of 3D Printing and Modeling to Aid in Neuroradiology Education for Medical Trainees
}

\author{
Michael A. Markovitz, MD; Sen Lu, MD; and Narayan A. Viswanadhan, MD
}

\begin{abstract}
Background: Applications of 3-dimensional (3D) printing in medical imaging and health care are expanding. Currently, primary uses involve presurgical planning and patient and medical trainee education. Neuroradiology is a complex subdiscipline of radiology that requires further training beyond radiology residency. This review seeks to explore the clinical value of 3D printing and modeling specifically in enhancing neuroradiology education for radiology physician residents and medical trainees.
\end{abstract}

Methods: A brief review summarizing the key steps from radiologic image to $3 \mathrm{D}$ printed model is provided, including storage of computed tomography and magnetic resonance imaging data as digital imaging and communications in medicine files; conversion to standard tessellation language (STL) format; manipulation of STL files in interactive medical image control system software (Materialise) to create 3D models; and 3D printing using various resins via a Formlabs 2 printer.

Results: For the purposes of demonstration and proof of concept, neuroanatomy models deemed crucial in early radiology education were created via open-source hardware designs under free or open licenses. 3D-printed objects included a sphenoid bone, cerebellum, skull base, middle ear labyrinth and ossicles, mandible, circle of Willis, carotid aneurysm, and lumbar spine using a combination of clear, white, and elastic resins.

Conclusions: Based on this single-institution experience, 3D-printed complex neuroanatomical structures seem feasible and may enhance resident education and patient safety. These same steps and principles may be applied to other subspecialties of radiology. Artificial intelligence also has the potential to advance the $3 \mathrm{D}$ process.
Michael Markovitz and Sen Lu are Radiology Resident Physicians at the University of South Florida in Tampa. Narayan Viswanadhan is Assistant Chief of Radiology at James A. Haley Veterans' Hospital in Tampa.

Correspondence:

Michael Markovitz (michaelmarkovitz@usf.edu)

Fed Pract. 2021;38(6). Published online June 10. doi:10.12788/fp.0134
A pplications of 3-dimensional (3D) printing in medical imaging and health care are expanding. 3D printing may serve a variety of roles and is used increasingly in the context of presurgical planning, as specific medical models may be created using individual patient imaging data. ${ }^{1}$ These patient-specific models may assist in medical trainee education, decrease operating room time, improve patient education for potential planned surgery, and guide clinicians for optimizing therapy. ${ }^{1,2}$ This article discusses the utility of 3D printing at a single institution to serve in enhancing specifically neuroradiology education.

\section{BACKGROUND}

As digital imaging and 3D printing have increased in popularity, the potential application of using imaging data to guide patient therapy has shown significant promise. Computed tomography (CT) is a commonly used modality that can be used to create 3D anatomical models, as it is frequently used in the medical setting, demonstrates excellent resolution to the millimeter scale, and can readily pinpoint pathology on imaging.

\section{Image Acquisition}

CT scans can be rapidly obtained, which adds significant value, particularly in the context of point-of-care 3D printing. Another modality commonly used for 3D printing is magnetic resonance imaging (MRI), which unlike CT, does not expose the patient to ionizing radiation. The $3 \mathrm{D}$ printing process is initiated with patient-specific CT or MRI data stored in the digital imaging and communications in medicine (DICOM) format, which is the international standard for communication and management of medical imaging information and related data. DICOM allows for faster and robust collaboration among imaging professionals. ${ }^{3}$

\section{Image Processing}

To print 3D anatomical models, patientspecific data must be converted from DICOM into standard tessellation language (STL) format, which can be created and edited with a variety of softwares. ${ }^{3}$ At James A. Haley Veterans' Hospital in Tampa, Florida, we use an image processing package that includes the Materialise 3-matic and interactive medical image control system. Image quality is essential; 


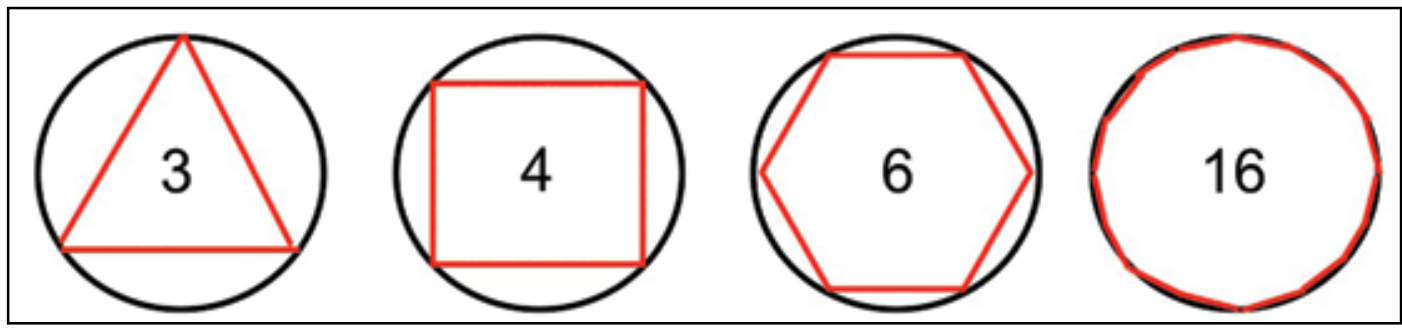

In 2 dimensions, the left 2 images demonstrate that 3 and 4 lines (red), respectively, poorly approximate a circle (black). On the right, increasing the number of lines to 6 and 16 better approximates the circle. Analogously, using many small triangles can create a high-quality model of complex 3 -dimensional human anatomy. ${ }^{5}$

therefore, careful attention to details such as pixel dimensions, slice thickness, and slice increments must be considered. ${ }^{3,4}$

An STL file creates a 3D image from triangle approximations. The entire 3D shape will be made of numerous large or small triangles, depending on the slice thickness, therefore, quality of the original radiologic image. The size and position of the triangles used to make the model can be varied to approximate the object's shape. The smaller the triangles, the better the image quality and vice versa. This concept is analogous to approximating a circle using straight lines of equal lengthmore, smaller lines will result in better approximation of a circle (Figure 1)., Similarly, using smaller triangles allows for better approximation of the image. As the human body is a complex structure, mimicking the body requires a system able to create nongeometrical shapes, which is made possible via these triangle approximations in a 3D STL file.

The creation of an STL file from DICOM data starts with a threshold-based segmentation process followed by additional finetuning and edits, and ends in the creation of a 3D part. The initial segmentation can be created with the threshold tool, using a Hounsfield unit range based on the area of interest desired (eg, bone, blood, fat). This is used to create an initial mask, which can be further optimized. The region grow tool allows the user to focus the segmentation by discarding areas that are not directly connected to the region of interest. In contrast, the split mask tool divides areas that are connected. Next, fine-tuning the segmentation using tools such as multiple slice edit helps to optimize the model. After all edits

\section{FIGURE 2 Correlation of the Sphenoid Bone Between} Computed Tomography and 3-Dimmensional Model

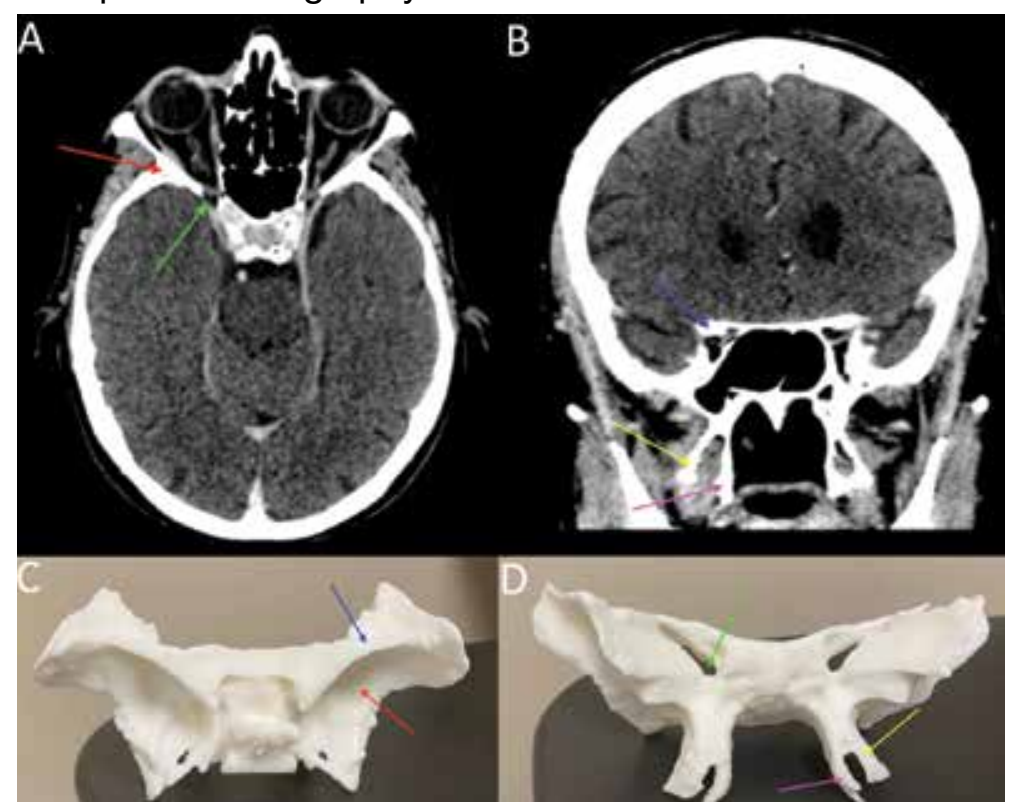

Axial (A) and coronal (B) head computed tomography scans depicting the sphenoid bone. The greater (red arrow) and lesser wings (blue arrow), medial (pink arrow) and lateral pterygoid plates (yellow arrow), and superior orbital fissure (green arrow) can be easily delineated on the superior $(C)$ and posterior (D) views of the sphenoid bone 3-dimensional model. ${ }^{7-9}$

are made, the calculate part tool converts the mask into a 3D component that can be used in downstream applications. For the purposes of demonstration and proof of concept, the models provided in this article were created via open-source hardware designs under free or open licenses..$^{7-9}$

\section{D Printing in Neuroradiology Education}

Neuroradiologists focus on diagnosing pathology related to the brain, head and neck, and spine. CT and MRI scans are the primary modalities used to diagnose these conditions. 3D printing is a useful tool for the trainee who wishes to fully understand 
FIGURE 3 Models of Complex Structures of the Head and Neck

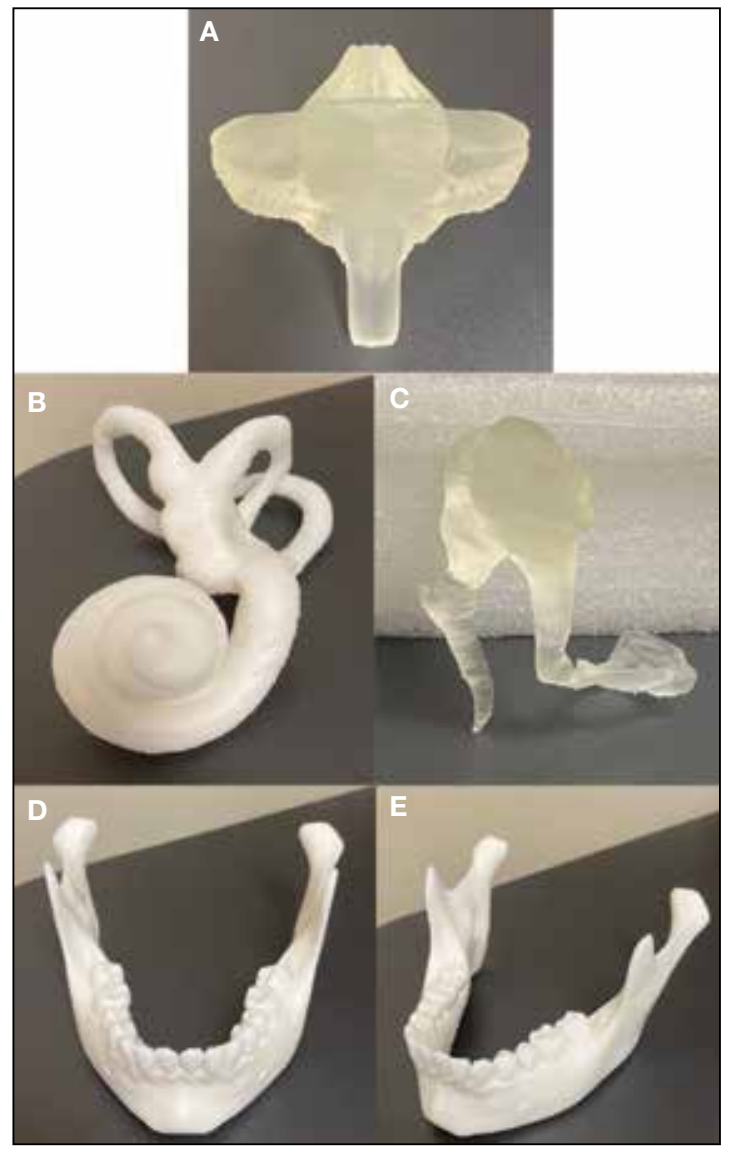

Formlabs 2 three-dimensional printer using standard tessellation language technology. $A$, resin models depict the anterior view of the cerebellum; $B$, middle ear bony and membranous labyrinth; $C$, ossicles; $\mathrm{D}$, anterior/superior view of the mandible; and $\mathrm{E}$, oblique view of the mandible. ${ }^{7-9}$

neuroanatomy and obtain further appreciation of imaging pathology as it relates to 3D anatomy. Head and neck imaging are a complex subdiscipline of neuroradiology that often require further training beyond radiology residency. A neuroradiology fellowship that focuses on head and neck imaging extends the training.

$3 \mathrm{D}$ printing has the potential to improve the understanding of various imaging pathologies by providing the trainee with a more in-depth appreciation of the anterior, middle, and posterior cranial fossa, the skull base foramina (ie, foramen ovale, spinosum, rotundum), and complex 3D areas, such as the pterygopalatine fossa, which are all critical areas to investigate on imaging. Figure 2 highlights how a complex anatomical structure, such as the sphenoid bone when printed in $3 \mathrm{D}$, can be correlated with CT cross-sectional images to supplement the educational experience.

Furthermore, the various lobes, sulci, and gyri of the brain and cerebellum and how they interrelate to nearby vasculature and bony structures can be difficult to conceptualize for early trainees. A 3Dprinted cerebellum and its relation to the brainstem is illustrated in Figure 3A. Additional complex head and neck structures of the middle ear membranous and bony labyrinth and ossicles and multiple views of the mandible are shown in Figures 3B through 3E.

3D printing in the context of neurovascular pathology holds great promise, particularly as these models may provide the trainee, patient, and proceduralist essential details such as appearance and morphology of an intracranial aneurysm, relationship and size of the neck of aneurysm, incorporation of vessels emanating from the aneurysmal sac, and details of the dome of the aneurysm. For example, the normal circle of Willis in Figure 4A is juxtaposed with an example of a saccular internal carotid artery aneurysm (Figure 4B).

A variety of conditions can affect the bony spine from degenerative, trauma, neoplastic, and inflammatory etiologies. A CT scan of the spine is readily used to detect these different conditions and often is used in the initial evaluation of trauma as indicated in the American College of Radiology appropriateness criteria. ${ }^{10}$ In addition, MRI is used to evaluate the spinal cord and to further define spinal stenosis as well as evaluate radiculopathy. An appreciation of the bony and soft tissue structures within the spine can be garnered with the use of 3D models (Figure 5).

Trainees can further their understanding of approaches in spinal procedures, including lumbar puncture, myelography, and facet injections. A variety of approaches to access the spinal canal have been documented, such as interspinous, paraspinous, and interlaminar oblique; 3D-printed models can aid in practicing these procedures. ${ }^{11}$ For example, a water-filled tube can be inserted into the vertebral canal to provide realistic tactile feedback for simulation of a lumbar 


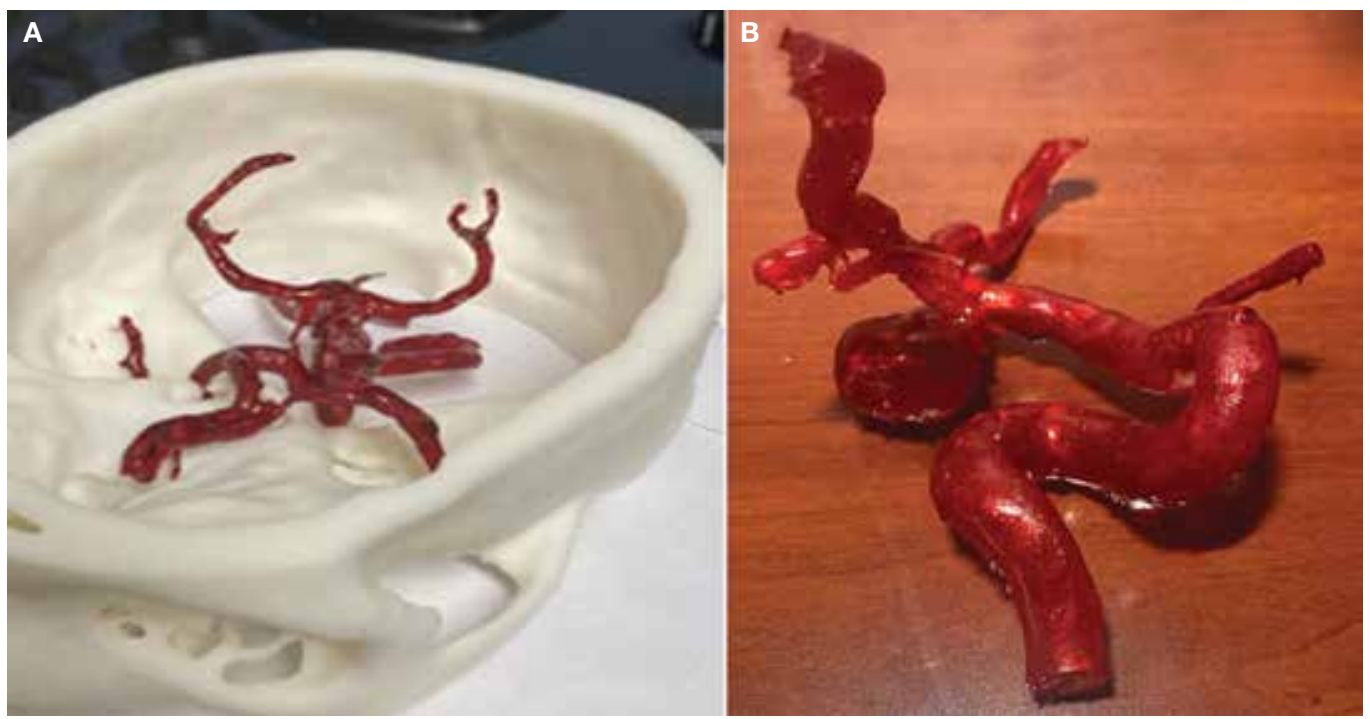

Formlabs 2 three-dimensional printer using standard tessellation language technology. A, elastic resins depict the normal circle of Willis, the anterior and posterior circulation is highlighted and its association to the anterior, middle, and posterior cranial fossa; B, the large outpouching with a narrow neck arising from the internal carotid artery consistent with an internal carotid artery aneurysm can be compared. ${ }^{7-9}$

puncture. An appreciation of the $3 \mathrm{D}$ anatomy can guide the clinician on the optimal approach, which can help limit time and potentially improve outcomes.

\section{Future Directions}

Artificial Intelligence (AI) offers the ability to teach computers to perform tasks that ordinarily require human intelligence. In the context of $3 \mathrm{D}$ printing, the ability to use AI to readily convert and process DICOM data into printable STL models holds significant promise. Currently, the manual conversion of a DICOM file into a segmented 3D model may take several days, necessitating a number FIGURE 5 Lumbar Spine 3-Dimensional Model

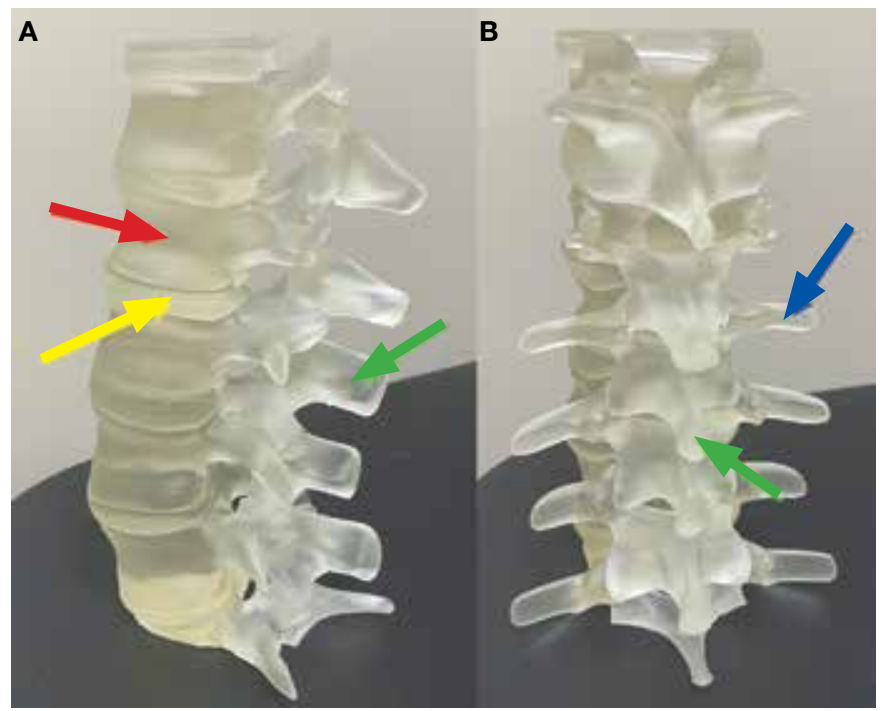

A, lateral; and B, posterior views of the lumbar spine. The vertebral bodies (red arrow), spinous (green arrow) and transverse processes (blue arrow), and intervertebral discs (yellow arrow) are all clearly demonstrated..$^{7-9}$ of productive hours even from the imaging and engineering champion. If machines could aid in this process, the ability to readily scale clinical 3D printing and promote widespread adoption would be feasible. Several studies already are looking into this concept to determine how deep learning networks may automatically recognize lesions on medical imaging to assist a human operator, potentially cutting hours from the clinical 3D printing workflow. ${ }^{12,13}$

Furthermore, there are several applications for $\mathrm{AI}$ in the context of 3D printing upstream or before the creation of a 3D model. A number of AI tools are already in use at the CT and MRI scanner. Current strategies leverage deep learning and advances in neural networks to improve image quality and 
create thin section DICOM data, which can be converted into printable 3D files. Additionally, the ability to automate tasks using AI can improve production capacity by assessing material costs and ensuring cost efficiency, which will be critical as point-of-care 3D printing develops widespread adoption. AI also can reduce printing errors by using automated adaptive feedback, using machine learning to search for possible print errors, and sending feedback to the computer to ensure appropriate settings (eg, temperature settings/environmental conditions)

\section{CONCLUSIONS}

Based on this single-institution experience, 3D-printed complex neuroanatomical structures seems feasible and may enhance resident education and patient safety. Interested trainees may have the opportunity to learn and be involved in the printing process of new and innovative ideas. Further studies may involve printing various pathologic processes and applying these same steps and principles to other subspecialties of radiology. Finally, AI has the potential to advance the $3 \mathrm{D}$ printing process in the future.

\section{Author disclosures}

The authors report no actual or potential conflicts of interest with regard to this article.

\section{Disclaimer}

The opinions expressed herein are those of the authors and do not necessarily reflect those of Federal Practitioner, Frontline Medical Communications Inc., the US Government, or any of its agencies.

\section{References}

1. Rengier F, Mehndiratta A, von Tengg-Kobligk H, et al. 3D printing based on imaging data: review of medical applications. Int J Comput Assist Radiol Surg. 2010;5(4):335-341. doi:10.1007/s11548-010-0476-x

2. Perica E, Sun Z. Patient-specific three-dimensional printing for pre-surgical planning in hepatocellular carcinoma treatment. Quant Imaging Med Surg. 2017;7(6):668-677. doi:10.21037/qims.2017.11.02

3. Hwang JJ, Jung Y-H, Cho B-H. The need for DICOM encapsulation of 3D scanning STL data. Imaging Sci Dent. 2018;48(4):301-302. doi:10.5624/isd.2018.48.4.301

4. Whyms BJ, Vorperian HK, Gentry LR, Schimek EM, Bersu ET, Chung MK. The effect of computed tomographic scanner parameters and 3-dimensional volume rendering techniques on the accuracy of linear, angular, and volumetric measurements of the mandible. Oral Surg Oral Med, Oral Pathol Oral Radiol. 2013;115(5):682691. doi:10.1016/j.00oo.2013.02.008

5. Materialise Cloud. Triangle reduction. Accessed May 20, 2021. https://cloud.materialise.com/tools/triangle -reduction

6. Comaneanu RM, Tarcolea M, Vlasceanu D, Cotrut MC. Virtual 3D reconstruction, diagnosis and surgical planning with Mimics software. Int J Nano Biomaterials. 2012;4(1);69-77.

7. Thingiverse: Digital designs for physical objects. Accessed May 20, 2021. https://www.thingiverse.com

8. Cults. Download for free 3D models for 3D printers. Accessed May 20, 2021. https://cults3d.com/en

9. yeggi. Search engine for $3 D$ printer models. Accessed May 20, 2021. https://www.yeggi.com

10. Expert Panel on Neurological Imaging and Musculoskeletal Imaging; Beckmann NM, West OC, Nunez $\mathrm{D}$, et al. ACR appropriateness criteria suspected spine trauma. J Am Coll Radiol. 2919;16(5):S264-285. doi:10.1016/j.jacr.2019.02.002

11. McKinney AM. Normal variants of the lumbar and sacra spine. In: Atlas of Head/Neck and Spine Normal Imaging Variants. Springer; 2018:263-321.

12. Sollini M, Bartoli F, Marciano A, et al. Artificial intelligence and hybrid imaging: the best match for personalized medicine in oncology. Eur J Hybrid Imaging. 2020;4(1):24. doi:10.1186/s41824-020-00094-8

13. Küstner T, Hepp T, Fischer M, et al. Fully automated and standardized segmentation of adipose tissue compartments via deep learning in 3D whole-body MRI of epidemiologic cohort studies. Radiol Artif Intell.2020;2(6):e200010. doi:10.1148/ryai.2020200010 